Annals of Pure and Applied Mathematics

Vol. 15, No. 1, 2017, 115-121

ISSN: 2279-087X (P), 2279-0888(online)

Published on 11 December 2017

www.researchmathsci.org

DOI: http://dx.doi.org/10.22457/apam.v15n1a11

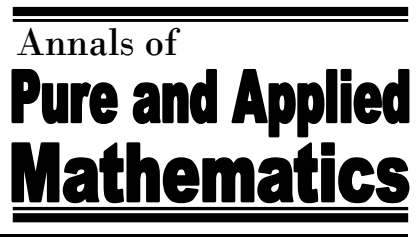

\title{
Split and Non-Split Domination Number in Bipolar Fuzzy Graphs
}

\author{
A. Prasanna ${ }^{1}$, C. Gurubaran ${ }^{2}$ and S. Ismail Mohideen ${ }^{3}$ \\ Department of Mathematics, Jamal Mohamed College (Autonomous) \\ Tiruchirappalli, Tamilnadu, India \\ ${ }^{1}$ E-mail: apj_jmc@yahoo.co.in; ${ }^{2} E$-mail: guruc2u@gmail.com; \\ ${ }^{3}$ E-mail: simohideen@yahoo.co.in
}

Received 15 November 2017; accepted 4 December 2017

Abstract. In this paper the concept of split domination and non - split domination in bipolar fuzzy graphs are introduced and investigated some of their properties. Also relationship between connected domination, split domination, strong split domination and non - split domination number in bipolar fuzzy graphs are discussed.

Keywords: Fuzzy graphs, Bipolar Fuzzy Graphs, Domination Number, Size of BFG, Order of BFG

AMS Mathematics Subject Classification (2010): 03E72, 05C72, 05C69

\section{Introduction}

A fuzzy set, introduced by Zadeh[8], gives the degree of membership of an object in a given set. Zhang [9] initiated the concept of a bipolar fuzzy set as a generalization of a fuzzy set. A bipolar fuzzy set is an extension of fuzzy set whose membership degree range is $[-1,1]$. In a bipolar fuzzy set, the membership degree 0 of an element means that the element is irrelevant to the corresponding property, and the membership degree $(0,1]$ of an element indicates that the element somewhat satisfies the property and the membership degree $[-1,0)$ of an element indicates that the element somewhat satisfies the implicit counter property. In 2011, Akram [1] introduced the concept of bipolar fuzzy graphs and defined different operations on it. Akram and Dudek [3] introduced the notions of regular bipolar fuzzy graphs.In 2013, Karunambigai, et. al., [4], introduced the concept of domination in bipolar fuzzy graphs.

In this paper, our aim is to introduce the concept of split domination and non split domination in Bipolar Fuzzy Graphs (BFG). Also we investigate relationship between connected domination, split domination, Strong Split domination and non - split domination in bipolar fuzzy graphs.

\section{Preliminaries}

In this section, we review some definitions that are necessary for this paper. 
A. Prasanna, C. Gurubaran and S. Ismail Mohideen

Definition 2.1. Let $X$ be a non - empty set. A bipolar fuzzy setA in $X$ is an object having the form $A=\left\{\left(x, \mu_{A}^{P}(x), \mu_{A}^{N}(x)\right) / x \in X\right\}$ where $\mu_{A}^{P}: X \rightarrow[0,1]$ and $\mu_{A}^{N}: X \rightarrow[-1,0]$ are mappings.

Definition 2.2. A Bipolar fuzzy graph $(B F G)$ is of the form $G=(V, E)$ where (i) $V=\left\{v_{1}, v_{2}, \ldots, v_{n}\right\}$ such that $\mu_{1}^{P}: X \rightarrow[0,1]$ and $\mu_{1}^{N}: X \rightarrow[-1,0]$ (ii) $E \subset V \times V$ where $\mu_{2}^{P}: V \times V \rightarrow[0,1]$ and $\mu_{2}^{N}: V \times V \rightarrow[-1,0]$ such that

$\mu_{2 i j}^{P}=\mu_{2}^{P}\left(v_{i}, v_{j}\right) \leq \min \left(\mu_{1}^{P}\left(v_{i}\right), \mu_{1}^{P}\left(v_{j}\right)\right)$ and

$\mu_{2 i j}^{N}=\mu_{2}^{N}\left(v_{i}, v_{j}\right) \geq \max \left(\mu_{1}^{N}\left(v_{i}\right), \mu_{1}^{N}\left(v_{j}\right)\right)$ for all $\left(v_{i}, v_{j}\right) \in E$.

Definition 2.3. A Bipolar fuzzy graph (BFG), $G=(V, E)$ is called strong if $\mu_{2}^{P}=$ $\min \left(\mu_{1}^{P}\left(v_{i}\right), \mu_{1}^{P}\left(v_{j}\right)\right)$ and $\mu_{2}^{N}=\max \left(\mu_{1}^{N}\left(v_{i}\right), \mu_{1}^{N}\left(v_{j}\right)\right)$ for all $\left(v_{i}, v_{j}\right) \in E$.

Definition 2.4. A Bipolar fuzzy graph (BFG), $G=(V, E)$ is called complete if $\mu_{2}^{P}=$ $\min \left(\mu_{1}^{P}\left(v_{i}\right), \mu_{1}^{P}\left(v_{j}\right)\right)$ and $\mu_{2}^{N}=\max \left(\mu_{1}^{N}\left(v_{i}\right), \mu_{1}^{N}\left(v_{j}\right)\right)$ for all $v_{i}, v_{j} \in V$.

Definition 2.5. Let $G=(V, E)$ be bipolar fuzzy graph (BFG), then the cardinality of $\mathrm{G}$ is defined to be $|G|=\sum_{v_{i} \in V}\left(\frac{1+\mu_{1}^{P}\left(v_{i}\right)+\mu_{1}^{N}\left(v_{i}\right)}{2}\right)+\sum_{\left(v_{i}, v_{j}\right) \in E}\left(\frac{1+\mu_{2}^{P}\left(v_{i}, v_{j}\right)+\mu_{2}^{N}\left(v_{i}, v_{j}\right)}{2}\right)$

Definition 2.6. Let $G=(V, E)$ be bipolar fuzzy graph (BFG), then the vertex cardinality of $\mathrm{G}$ is defined to be $|V|=\sum_{v_{i} \in V}\left(\frac{1+\mu_{1}^{P}\left(v_{i}\right)+\mu_{1}^{N}\left(v_{i}\right)}{2}\right)$ for all $v_{i} \in V$

Definition 2.7. Let $G=(V, E)$ be bipolar fuzzy graph (BFG), then the edge cardinality of $\mathrm{G}$ is defined to be $|E|=\sum_{\left(v_{i}, v_{j}\right) \in E}\left(\frac{1+\mu_{2}^{P}\left(v_{i}, v_{j}\right)+\mu_{2}^{N}\left(v_{i}, v_{j}\right)}{2}\right)$ for all $\left(v_{i}, v_{j}\right) \in E$

Definition 2.8. The degree of a vertexv in a BFG, $G=(V, E)$ is defined to be sum of the weights of the strong edges incident at $v$. It is denoted by $d_{G}(v)$

Definition 2.9. The minimum degree of $G$ is $\delta(G)=\min \left\{d_{G}(v) \mid v \in V\right\}$ The maximum degree of $G$ is $\Delta(G)=\max \left\{d_{G}(v) \mid v \in V\right\}$.

Definition 2.10. An edge (u, v) is said to be strong edge in BFG, $G=(V, E)$ if $\mu_{2}^{P}\left(v_{i}, v_{j}\right) \geq\left(\mu_{2}^{P}\right)^{\infty}\left(v_{i}, v_{j}\right)$ and $\mu_{2}^{N}\left(v_{i}, v_{j}\right) \leq\left(\mu_{2}^{N}\right)^{\infty}\left(v_{i}, v_{j}\right)$.

Where $\left(\mu_{2}^{P}\right)^{\infty}\left(v_{i}, v_{j}\right)=\max \left\{\left(\mu_{2}^{P}\right)^{k}\left(v_{i}, v_{j}\right) \mid k=1,2,3 \ldots, n\right\}$ and $\left(\mu_{2}^{N}\right)^{\infty}\left(v_{i}, v_{j}\right)=\min \left\{\left(\mu_{2}^{N}\right)^{k}\left(v_{i}, v_{j}\right) \mid k=1,2,3 \ldots, n\right\}$.

Let $v_{i}$ be a vertex in a BFG $G=(V, E)$, then of $u$ in $G$.

$$
N(u)=\left\{v: v \in V \text { and }\left(v_{i}, v_{j}\right) \text { is a strong edge in } G\right\} \text { is called neighbourhood }
$$


Split and Non-Split Domination Number in Bipolar Fuzzy Graphs

Definition 2.11. Let $G=(V, E)$ be a bipolar fuzzy graph, if all the vertices have the same open neighbourhood degree $n$, then $G$ is called $\mathrm{n}$ - regular bipolar fuzzy graph.

Definition 2.12. Let $G=(V, E)$ be a bipolar fuzzy graph, if each vertex of $G$ has same close neighbourhooddegree $m$, then $G$ is called a totally regular bipolar fuzzy graph. The closed neighbourhood degree of a vertex $v$ is defined by $\operatorname{deg}^{P}[v]=\operatorname{deg}^{P}(v)+$ $\mu_{A}^{P}(v)$ and $\operatorname{deg}^{N}[v]=\operatorname{deg}^{N}(v)+\mu_{A}^{N}(v)$.

Definition 2.13. Two vertices in a $\mathrm{BFG}, G=(V, E)$ are said to be independent if there is no strong edge between them.

A subset $S$ of $V$ is said to be independent set if $\mu_{2}^{P}\left(v_{i}, v_{j}\right)<\left(\mu_{2}^{P}\right)^{\infty}\left(v_{i}, v_{j}\right)$ and $\mu_{2}^{N}\left(v_{i}, v_{j}\right)>\left(\mu_{2}^{N}\right)^{\infty}\left(v_{i}, v_{j}\right)$ for all $v_{i}, v_{j} \in S$

An independent set $S$ of BFG $G=(V, E)$ is said to be maximal independent, if for every vertex $v \in V-S$ the set $S \cup\{v\}$ is not independent.

Definition 2.14. A subset $D$ of $V$ is called a dominating set in $G$ if for every $v \in V-D$, there exist $u \in D$ such that $u$ dominates $v$. A dominating set $D$ of a BFG is said to be minimal dominating set if no proper subset of $D$ is a dominating set. Minimum cardinality among all minimal dominating set is called lower domination number of $G$, and is denoted by $d_{B}(G)$. Maximum cardinality among all minimal dominating set is called upper domination numberof $G$, and is denoted by $D_{B}(G)$.

\section{Split, non - split and connected domination}

Definition 3.1. A dominating set $D$ of a bipolar fuzzy graph $G$ is a split dominating set if the induced bipolar fuzzy subgraph $\langle V-D\rangle$ is disconnected.

Definition 3.2. A dominating set $D$ of a bipolar fuzzy graph $G=\langle V, E\rangle$ is a strong split dominating set if the induced bipolar fuzzy subgraph $\langle V-D\rangle$ is totally disconnected with atleast two components.

Definition 3.3. The split domination number of a bipolar fuzzy graph $G$ is $\gamma_{S}(G)$ is the minimum bipolar fuzzy cardinality of a split dominating set.

Definition 3.4. The strong split domination number of a bipolar fuzzy graph $G$ is $\gamma_{S S}(G)$ is the minimum bipolar fuzzy cardinality of a strong split dominating set.

Definition 3.5. A dominating set $D$ of a bipolar fuzzy graph $G$ is a connected dominating set if the induced bipolar fuzzy subgraph $\langle D\rangle$ is connected.

Definition 3.6. The connected domination number of a bipolar fuzzy graph $G$ is $\gamma_{C}(G)$ is the minimum bipolar fuzzy cardinality of a connected dominating set.

Definition 3.7. A dominating set $D$ of a bipolar fuzzy graph $G$ is a non-split dominating set if the induced bipolar fuzzy subgraph $\langle\mathrm{V}-\mathrm{D}\rangle$ is connected. 


\section{A. Prasanna, C. Gurubaran and S. Ismail Mohideen}

Definition 3.8. The non-split domination number of a bipolar fuzzy graph $G$ is $\gamma_{n s}(G)$ is the minimum bipolar fuzzy cardinality of a non-split dominating set.

Example 3.9. Consider the Bipolar Fuzzy Graph $(B F G), G=(V, E)$ six vertices and nine edges.

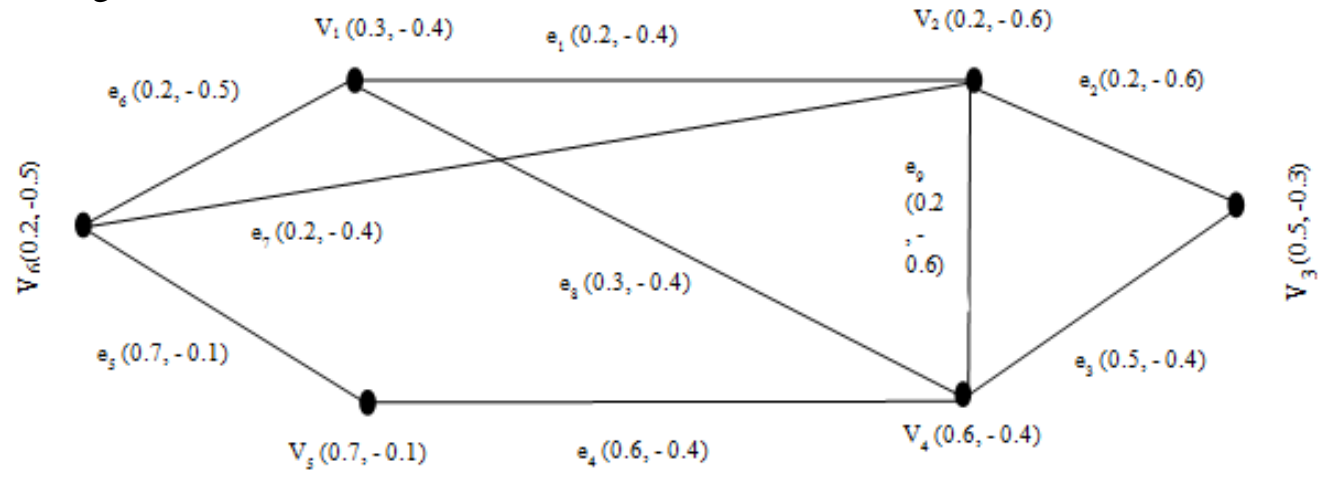

In BFG,

Figure 1:

Split Dominating Set $D_{1}=\left\{v_{1}, v_{3}, v_{5}\right\}$ Cardinality of Set $\left|D_{1}\right|=1.25$

Non - Split Dominating Set $D_{2}=\left\{v_{1}, v_{4}, v_{3}\right\}$ Cardinality of Set $\left|D_{2}\right|=1.45$

Connected Dominating Set $D_{3}=\left\{v_{1}, v_{2}, v_{4}\right\}$ Cardinality of Set $\left|D_{3}\right|=1.35$

Proposition 3.10. A dominating set $D$ of a bipolar fuzzy graph $G$ is a strong split dominating set iff there exist two bipolar fuzzy vertices $v_{i}, v_{j} \in V-D$ such that every $v_{i}-v_{j}$ path contains a bipolar fuzzy vertex of $D$.

Proof:

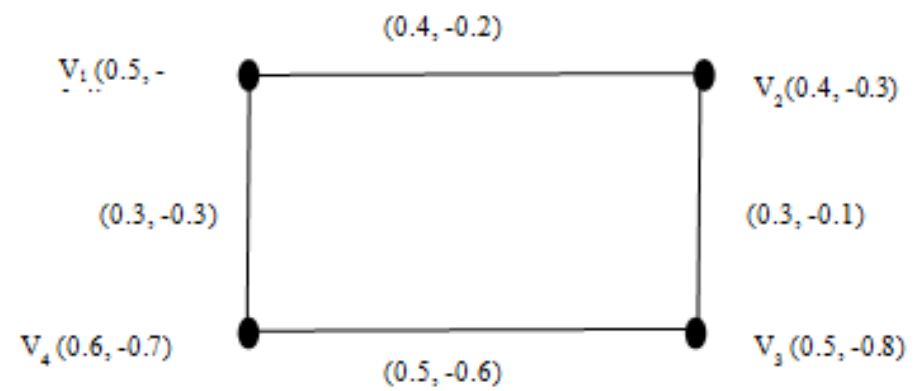

Figure 2:

$\langle\mathrm{V}-\mathrm{D}\rangle$ is a bipolar fuzzy graph of $\mathrm{G}$ induced by totally disconnected, hence $\mathrm{D}$ is a strong split dominating set of $G$ with strong split domination number $\gamma_{S S}(G)$ we see that there exists $\mathrm{v}_{2} \mathrm{v}_{4} \in \mathrm{V}-\mathrm{D}$ such that $\mathrm{v}_{1}-\mathrm{v}_{3}$ path contains $\mathrm{v}_{2}$.

Proposition 3.11. A split dominating set $D$ of a Bipolar fuzzy graph $G$ is minimal for each vertex $v \in D$, one of the following three conditions holds

i) $\quad$ There exist $u \in V-D$ such $N(u) \cap D=\{v\}$

ii) $\quad \mathrm{v}$ is isolated vertex in $\langle D\rangle$

iii) $\quad<(V-D) \cup\{v\}>$ is connected 
Split and Non-Split Domination Number in Bipolar Fuzzy Graphs

Proof: Suppose $D$ is minimal and there exist a vertex $v \in D$ such that $v$ does not hold any of the above conditions. Then by conditions (i) and (ii) $D_{1}=D-\{v\}$ is a dominating set of $G$. also by (iii), $\langle V-D\rangle$ is split dominating set of $G$, a contradiction.

Proposition 3.12. For any bipolar fuzzy graph $G=\left\{\sigma, \mu^{P}, \mu^{N}\right\}$ then $\gamma(G) \leq \gamma_{S S}(G)$.

Proof: It's obviously true by the definitions

Proposition 3.13. For any regular bipolar fuzzy graph the split domination number is same for all the minimal dominating set.

Proof: By the definition, regular BFG all the vertices have the same vertex cardinality. This shows that every dominating set which in minimal same domination number in BFG.

Proposition 3.14. In total regular bipolar fuzzy graph $\gamma_{n s}(G)=\gamma_{C}(G)$

Proposition 3.15. In total regular bipolar fuzzy graph $\gamma_{\mathrm{ns}}(\mathrm{G})=\min \left\{|\mathrm{V}|_{\mathrm{v}_{\mathrm{i}} \in \mathrm{G}}\right\}$

Proposition 3.16. In a bipolar fuzzy graph both regular and totally regular, then $\gamma_{\mathrm{ns}}(\mathrm{G})$ is constant for all minimal dominating set.

Proposition 3.17. If $G$ be a regular bipolar fuzzy graph, where crisp graph $G^{*}$ is an odd cycle then $\gamma_{\mathrm{ns}}(\mathrm{G})$ is constant.

Proposition 3.18. For any Bipolar fuzzy graph $G$ with an end vertex $\gamma(G)=\gamma_{S}(G)$. Furthermore, there exist a $\gamma_{S}$-set of $G$ containing all vertices adjacent to end vertices.

Proof: Let $v$ be an end vertex of $G$, then there exist a cut vertex $w$ adjacent to $v$. Let $D$ be a $\gamma_{S}$-set of G suppose $w \in D$, then is a $\gamma_{S}$-set ofG suppose $w \in V-D$ then $v \in D$ and hence $(D-\{v\}) \cup\{w\}$ is a $\gamma_{S}-$ set ofG.

Repeating this process for all such cut vertices adjacent to end vertices we obtain a $\gamma_{S}-$ set of $G$ containing all cut vertices adjacent to end vertices.

Proposition 3.19. For any bipolar fuzzy graph (BFG) $G=\left(\sigma, \mu^{\mathrm{P}}, \mu^{\mathrm{N}}\right), \gamma_{\mathrm{ss}}(\mathrm{G}) \leq$ p. $\Delta(\mathrm{G}) /(\Delta(\mathrm{G})+1)$.

Proof: Let $\mathrm{D}$ be a split dominating set since $\mathrm{D}$ is minimal, by Proposition it follows that for each $\mathrm{v} \in \mathrm{D}$ there exist $\mathrm{u} \in \mathrm{V}-\mathrm{D}$ such that $0<\mu(\mathrm{u}, \mathrm{v})=\sigma(\mathrm{u}) \wedge \sigma(\mathrm{v})$, $\mathrm{v}$ is adjacent to $\mathrm{u}$. which implies that $\mathrm{V}-\mathrm{D}$ is a dominating set of $\mathrm{G}$.

Thus $\gamma(G) \leq|V-D| \leq p-\gamma_{s s}(G)$ and by,For any BFG, $G=\left(\sigma, \mu^{P}, \mu^{N}\right)$

Hence $\gamma_{\mathrm{ss}}(\mathrm{G}) \leq$ p. $\Delta(\mathrm{G}) /(\Delta(\mathrm{G})+1)$.

$$
\gamma(\mathrm{G}) \geq \mathrm{p} /(\Delta(\mathrm{G})+1)
$$

Definition 3.20. A set of Bipolar fuzzy vertex which cover all the Bipolar fuzzy edges is called a bipolar fuzzy vertex cover of $G$ and minimum cardinality of a bipolar fuzzy vertex cover is called a vertex covering number of $G$ and denoted by $\beta(G)$. 


\section{A. Prasanna, C. Gurubaran and S. Ismail Mohideen}

Definition 3.21. A disconnection of a bipolar fuzzy graph $G$ is a vertex set $V$ whose removal results in a disconnected or a single vertex graph. The weight of $\mathrm{V}$ is a defined to $\sum\left\{\min \left(\mu^{\mathrm{P}}\left(\mathrm{v}_{1}, \mathrm{v}_{2}\right)\right), \min \left(\mu^{\mathrm{N}}\left(\mathrm{v}_{1}, \mathrm{v}_{2}\right)\right) / \mu^{\mathrm{P}}\left(\mathrm{v}_{1}, \mathrm{v}_{2}\right)\right.$ or $\left.\mu^{\mathrm{N}}\left(\mathrm{v}_{1}, \mathrm{v}_{2}\right), \mathrm{u} \in \mathrm{V}, \mathrm{v} \in \mathrm{D}\right\} \quad$ the vertex connectivity of bipolar fuzzy graph $G$ denoted by $\Omega(G)$ is defined to be the minimum weight disconnection inG.

Proposition 3.22. For any BFG G $=\left(\sigma, \mu^{\mathrm{P}}, \mu^{\mathrm{N}}\right)$, (i) $\gamma(\mathrm{G}) \leq \gamma_{\mathrm{ss}}(\mathrm{G})$ (ii) $\Omega(\mathrm{G}) \leq \gamma_{\mathrm{ss}}(\mathrm{G})$ Proof:

(i) and (ii) follows from the definitions of $\gamma(\mathrm{G}), \gamma_{\mathrm{ss}}(\mathrm{G})$ and $\Omega(\mathrm{G})$

Proposition 3.23. For any bipolar fuzzy graph (BFG) $G=\left(\sigma, \mu^{\mathrm{P}}, \mu^{\mathrm{N}}\right), \gamma_{\mathrm{ss}}(\mathrm{G}) \leq \beta(\mathrm{G})$

Proof: Let D be a maximal independent set of bipolar fuzzy vertex in $G$, then $G$ has atleast two bipolar fuzzy vertices and every bipolar fuzzy vertex in $D$ is adjacent to some vertex in $\mathrm{V}-\mathrm{D}$. this implies that $\mathrm{V}-\mathrm{D}$ strong split dominating set of $\mathrm{G}$. Thus the Proposition holds.

Proposition 3.24. For any bipolar fuzzy graph (BFG) $G=\left(\sigma, \mu^{\mathrm{P}}, \mu^{\mathrm{N}}\right) \gamma_{\mathrm{s}}(\mathrm{G}) \leq \alpha_{0}(\mathrm{G})$

Proof: Let $S$ be minimum independent set of vertices in $G$. Then $S$ has atleast two vertices in $G$. Then $S$ has atleast two vertices and every vertex in $S$ has effective edge to some vertex in $\mathrm{S}$ has effective edge to some vertex in $\mathrm{V}-\mathrm{S}$. this shows that $\mathrm{V}-\mathrm{Sa}$ split dominating set of $G$. Hence $\gamma_{s}(G) \leq \alpha_{0}(G)$.

Proposition 3.25. For any bipolar fuzzy graph (BFG) $G=\left(\sigma, \mu^{\mathrm{P}}, \mu^{\mathrm{N}}\right) \gamma(\mathrm{G})+\gamma_{\mathrm{s}}(\mathrm{G}) \leq$ $2|v|$.

Proof: Let D be a minimal dominating set of BFG in G, D contains the vertices with minimum degree then $\mathrm{D}$ be split domination number is same for the minimum degree vertices in $\mathrm{G}$. here domination number and split domination number lesser values of vertex covering and minimum weight of disconnection in $\mathrm{G}$.

$$
\beta(G)+\alpha_{0}(G) \leq 2|v|
$$

Here $|\mathrm{v}|$ denotes the vertex cardinality will be minimum in the set $\mathrm{V}$.

Proposition 3.26. If $\gamma_{s s}(G) \leq \gamma_{c}(G)$ then for any strong split dominating set

Proof: Since D is minimal, V - D is dominating set of $\mathrm{G}$ and furthermore it is strong split dominating set since $\langle\mathrm{D}\rangle$ is totally disconnected.

\section{Conclusion}

In this paper we introduced the concept of some domination numbers.Alsorelationship between connected domination and split domination number in bipolar fuzzy graphs are discussed.

\section{REFERENCES}

1. M.Akram, Bipolar fuzzy graphs, Information Sciences, 181 (2011) 5548 - 5564.

2. M.Akram, Bipolar fuzzy graphs with applications, Knowledge - Based systems 39 (2013) 1-8. 
Split and Non-Split Domination Number in Bipolar Fuzzy Graphs

3. M.Akram and Wieslaw A. Dudek, Regular bipolar fuzzy graphs, Neural Comput. andApplic., 21 (Suppl 1) (2012) S197 - S205.

4. M.G.Karunambigai, M.Akram, K.Palanivel and S.Sivasankar, Domination in bipolar fuzzy graphs, Proceedings of the international conference on fuzzy systems, FUZZ IEEE (2013) 1-6.

5. V.Mohanaselvi and S.Sivamani, Paramount domination in bipolar fuzzy graphs, Int. Journal of Sci. Engg. Research, 7(5) (2016) 50 - 52.

6. A.NagoorGani, B.Ahamed, Order and size in fuzzy graphs, Bulletin of Pure and Applied Sciences, 22E (1) (2003) 145 - 148.

7. H.Yang, S.Li, W.Yang and Y.Lu, Notes on bipolar fuzzy graphs, Information Sciences, 242 (2013) 113-121.

8. L.A.Zadeh, Fuzzy sets, Information and Control, 8 (1965) 338-353.

9. W.R.Zhang, Bipolar fuzzy sets and relations: a computational framework for cognitive modeling and multiagent decision analysis, Industrial Fuzzy Control and Intelligent Systems Conference, and the NASA Joint Technology Workshop on Neural Networks and Fuzzy Logic, (1994) 305-309.

10. S.Samanta and M.Pal, Irregular bipolar fuzzy graphs, International Journal of Applications of Fuzzy Sets, 2 (2012) 91-102.

11. S.Samanta and M.Pal, Bipolar fuzzy hypergraphs, International Journal of Fuzzy Logic Systems, 2(1) (2012) 17-28.

12. H.Rashmanlou and M.Pal, Some properties of highly irregular interval valued fuzzy graphs, World Applied Sciences Journal, 27(12) (2013) 1756-1773.

13. S.Samanta and M.Pal, Some more results on bipolar fuzzy sets and bipolar fuzzy intersection graphs, The Journal of Fuzzy Mathematics, 22(2) (2014) 253-262.

14. H.Rashmanlou, S.Samanta, M.Pal, R.A.Borzooei, A study on bipolar fuzzy graphs, Journal of Intelligent and Fuzzy Systems, 28 (2015) 571-580.

15. H.Rashmanlou, S.Samanta, M.Pal and R.A.Borzooei, Bipolar fuzzy graphs with Categorical properties, The International Journal of Computational Intelligence Systems, 8(5) (2015) 808-818.

16. H.Rashmanlou, S.Samanta, M.Pal and R.A.Borzooei, Product of bipolar fuzzy graphs and their degree, International Journal of General Systems, 45(1) (2016) 1-14.

17. G.Ghorai and M.Pal, Certain types of product bipolar fuzzy graphs, Intern. J. Applied and Computational Mathematics, 3(2) (2017) 605-619. 